\title{
An audit to evaluate the effect of staff training on the use of the malnutrition universal screening tool (MUST)
}

\author{
S. Wong \\ Department of Nutrition and Dietetics, Stoke Mandeville Hospital, Aylesbury, UK
}

\begin{abstract}
Malnutrition is frequently reported in hospitalised adults and it is associated with adverse hospital outcomes. Patients admitted to hospital should be routinely screened and monitored regularly ${ }^{(1)}$. The malnutrition universal screening tool (MUST) ${ }^{(2)}$ has been recommended for nutrition screening since 2004. An audit was carried out in 2005 to assess the use of the MUST in Buckinghamshire Hospitals NHS Trust (BHT) and found that the use was low. Regular training on nutritional screening to ward staff has been given by ward dietitians for the last 2 years.

The aims of the present audit were to assess the use of the MUST in district general hospitals and to find out if there has been any improvement after 2 years of implementation. This audit was carried out from May to June 2007 in three hospital sites (Stoke Mandeville Hospital (SMH), Wycombe General Hospital (WGH) and Amersham Hospital (AH)) in BHT. Data were collected by ward dietitians from individual patient notes using a standard audit questionnaire.

A total of 432 sets of notes were examined (SMH, 212; WGH + AH, 220). It was found that $288(67 \%)$ sets of notes had a completed MUST form (SMH, 125 (59\%); WGH + AH, 163 (74\%)). In the 2005 audit 54\% of patients admitted to BHT were screened using MUST (SMH, 71 (39\%); WGH + AH, $141(67 \%)$ ). The current audit showed a significant improvement in the use of MUST in BHT over the previous 2 years $(54 \%$ in 2005 v. $67 \%$ in $2007 ; P<0.001)$.

Since the audit in 2005 efforts have been successful in improving compliance in screening with the MUST. In order to enhance compliance in nutrition screening, training on the use of MUST to all clinical staff is now included as a part of the BHT induction programme. In addition, a BHT nutrition committee has been set up to ensure nutrition screening as a priority in the BHT. The present audit has shown that the compliance of completing MUST has improved over the last 2 years. Further training and updates on nutrition screening to encourage ward staff to use and complete the MUST and take responsibility for nutrition is critical. A series of action plans including a call for a review of BHT nutrition screening policy, engagement of nursing management and adaptation of nutrition into the admission pack for all hospital inpatients have been agreed following the present audit.
\end{abstract}

1. National Institute for Health and Clinical Excellence (2006) Nutrition Support in Adults: Oral Nutrition Support, Enteral Tube Feeding And Parenteral Nutrition. London: NICE.

2. Elia M (2003) Screening for Malnutrition: A Multidisciplinary Responsibility. Development and Use of the Malnutrition Universal Screening Tool (MUST) for Adults. Redditch, Worcs.: BAPEN. 